\title{
Development of an Indirect Enzyme-Linked Immunosorbent Assay Using Recombinant Microneme 10 (MIC10) Protein of Toxoplasma gondii for Diagnostic Evaluation in Murine Model Infected with Tachyzoites
}

\author{
Raghubir Singh $^{1 *}$, M.A. Malik ${ }^{1}$, M. Sankar ${ }^{2}$, Maninder Singh', \\ H.K. Sharma ${ }^{1}$ and K. Sahil ${ }^{3}$ \\ ${ }^{1}$ Division of Veterinary Public Health and Epidemiology, F.V.Sc \& A.H, R.S. Pura, \\ SKUAST-J, Jammu, India \\ ${ }^{2}$ Temperate Animal Husbandry, Indian Veterinary Research Institute, Mukteshwar, \\ Nainital, India \\ ${ }^{3}$ Division of Livestock Production and Management, F.V.Sc \& A.H, R.S.Pura, SKUAST-J, \\ Jammu, India \\ *Corresponding author
}

\section{A B S T R A C T}

Toxoplasma gondi being microscopic size and obligate intracellular, its proliferative forms cultivation in the laboratory is relative difficult and thus, immunodiagnostic tools such as

Keywords

Toxoplasma, MIC10, ELISA, Sensitivity, Specificity

Article Info

Accepted:

04 February 2018

Available Online:

10 March 2018 serology and immune-histochemistry development becomes essential to demonstrate the infection in vivo. Among the various existing serological methods, the enzyme-linked immunosorbent assay (ELISA) is simple, rapid and economical, rendering it suitable for clinical diagnosis applications. The aim of present study was to express the microneme 10 (MIC10) antigenic protein which is a circulating antigen following invasion by tachyzoites in hosts in the prokaryotic expression system and evaluated as a potential diagnostic marker by indirect ELISA using the infected and non infected sera samples from Swiss albino mice. Optimum working dilutions of the antigen, sera and the conjugate were determined by checker board titrations and the cut off value was generated as mean plus standard deviation of optical densities of negative sera by ELISA. Recombinant MIC10ELISA at the cut off value of 1.2539 showed the sensitivity and specificity as $79.2 \%$ and $87.2 \%$ respectively. The data was generated as a step to evaluate the potential of MIC10 antigen protein as a diagnostic marker.

\section{Introduction}

Toxoplasmosis is a widely prevalent infection in the warm blooded animals including human beings has the causative agent Toxoplasma gondii. Among TORCH (Toxoplasmosis,
Rubella, Cytomegalovirus and Herpes simplex virus) group of infection, this disease may be acquired either during pregnancy or postnatal life in humans (Haddadzadeh et al., 2006). Toxoplasma gondii, type I (RH) strain is a major opportunistic pathogen in the immune- 
compromised patients causing atypical ocular toxoplasmosis and fatal encephalitis (Grigg and Boothroyd, 2001). Conventionally, diagnosis of toxoplasmosis is mainly based on the demonstration of the parasites in situ or in biological materials in acute cases and by application of serological tools for indirect detection in chronic or latent infections. These methods are time consuming and often subject to the limitations of sensitivity and specificity. The commonly used agglutination test, viz. modified agglutination test and dye test require handling of live tachyzoites and there is a potential risk of infection to human handlers. Serological tests have been widely applied and accepted for $T$. gondii infections diagnosis based on the detection of $\operatorname{IgG}$ antibodies in humans and animals (Kotresha and Noordin, 2010; Holec-Gasior, 2013).

Among the various existing serologic methods, the enzyme-linked immunosorbent assay (ELISA) is simple, rapid and economical, rendering it suitable for clinical diagnosis applications. Compared with other detection methods such as PCR and indirect fluorescence antibody test (IFAT) which requires special equipment, ELISA is much cheaper and easier. Since a number of the serological targets for the detection of $T$. gondii antibodies are sequestered within internal organelles, the method of preparation of the tachyzoite antigen has a significant effect on assay performance, as shown by the differential agglutination assay (Dannemann $e t$ al., 1990).

The recombinant proteins which are presumed to be the candidates for diagnosis and used for subunit vaccine development are surface antigen (SAG), micronemes (MIC), rhoptries (ROP), and dense granules (GRA). Aubert et al., (2000) evaluated the diagnostic utility of eleven $T$. gondii recombinant antigens viz., P22 (SAG-2), P24 (GRA-1), P25, P28 (GRA2), P29 (GRA-7), P30 (SAG-1), P35, P41
(GRA-4), P54 (ROP-2), P66 (ROP-1), and $\mathrm{P} 68$ in $\operatorname{IgG}$ and $\operatorname{IgM}$ recombinant enzyme linked immunosorbent assays (Rec-ELISAs). The SAG-1 antigen had been the focus of intensive research with the aim of developing a diagnostic tool against acute $T$. gondii infection (Marti et al., 2001). In an attempt to develop a recombinant protein based ELISA, the gene coding SAG-1 of $T$. gondii was cloned and expressed in E. coli as glutathioneS-transferase (GST) fusion protein by Kimbita et al., (2001). The recombinant SAG-1 (rSAG-1) was used in the ELISA and the specificity of the recombinant protein was confirmed by Western blotting. The diagnostic efficiency of the recombinant proteins SAG-1 and GRA-7, either individually or as a cocktail of the recombinant proteins, was assessed with 56 reference goat sera by ELISA (Velmurugan et al., 2008). The immunoreactivity of the refolded SAG-1 and GRA-7 was evidenced by high O.D. values. The reactivity of the recombinant proteins as a cocktail preparation was more than that of individual proteins in ELISA and could detect accurately the infection in goats. The performance of the four fusion proteins GST-GRA-3, GST-GRA-7, GST-MIC-3 and D-SAG-1 was assessed using ELISA with the 60 IgG-positive samples, and for each recombinant product the cut off value was determined as the mean plus 2 fold standard deviations (SD) of the absorbance readings obtained for the 30 Toxoplasma IgGnegative sera. D-SAG-1 and GST-MIC-3 fusion proteins reacted with 92 and $95 \%$ of the positive sera, respectively, and both GSTGRA-3 and GST-GRA-7 reacted with $85 \%$ of the positive sera (Beghetto et al., 2003).

Another solution to preliminary validation of these Rec-ELISA may be the utilization of systematically collected sera obtained from experimentally infected animals. Thus, in the present study a new antigenic target microneme 10 (MIC 10) was selected for in vitro expression and use of it in Rec-ELISA to 
evaluate its diagnostic potential with the systematically collected animal sera from two groups of Swiss albino mice each with forty animals and a group animals were infected with tachyzoites where as other group was kept as control.

Microneme 10 (MIC10) lacks the putative adhesive and transmembrane domain that is capable of binding to host cell receptor(s) and hence this raises the possibility that MIC10 could diffuse from the infection site in the tissue and become accessible as a circulating antigen (Hoff et al., 2001). Further, molecular characterization of a full-length MIC10 cDNA revealed that MIC10 lacks homology to any previously characterized proteins, although a homologue, NcMIC10, was identified in a closely related parasite, $N$. caninum. MIC10 gene possesses nine di-glutamic acid repeats and an imperfect repeat sequence $(\mathrm{RK}(\mathrm{R} / \mathrm{Y})$ HEEL), and is entirely devoid of cysteines. Antibodies against recombinant MIC10 recognized the native MIC10 and localized the protein to the micronemes in indirect immunofluorescence and immuno electromicroscopic experiments. Comparison of immunofluorescence images indicates that MIC10 expression is higher in $T$. gondii tachyzoites than in bradyzoites and was shown to be released from parasites (Zhou et al., 2005). The results demonstrated that MIC10 may be a promising serodiagnostic marker for $T$. gondii infection and may provide the foundation alone or with other antigenic markers for the development of reliable and standard diagnostic methods in other host species including farm animals and humans.

\section{Materials and Methods}

\section{Parasite}

The Toxoplasma gondii RH strain tachyzoites were procured from the Department of Medical Parasitology, Post-graduation institute of medical education and research (PGIMER), Chandigarh.

\section{Experimental animals}

Inbred Swiss albino mice of either sex of 6-12 weeks of age, weighing about 25-30 gm were used in the study. The mice were maintained under standard rearing conditions in the divisional laboratory animal shed. All the experimental procedures were carried out with prior permission of the institutional animal ethics committee (IAEC).

\section{Infection and collection of mice sera}

A total number of 80 adult Swiss albino mice of either sex were divided into two groups of 40 each. Group A mice were inoculated with $1 \times 10^{2}$ tachyzoites of mouse adopted $T$. gondii RH strain. Between day 6 to 7 post-infection (PI), all the mice showed symptoms of Toxoplasma infection with a mortality in 16 mice of group A due to infection. The group B mice were kept as control and were without infection with death of one mouse by day 6 . The sera were collected from both the infected and control group mice from the tail vein and the inner canthus of the eye after days 6 to 7 PI. The sera were available from 24 infected mice and 39 control group mice for serodiagnosis.

\section{Expression and purification of recombinant MIC10}

Based on the nucleotide sequence of MIC10 (GENBANK accession number AF293654), the PCR primers for amplification of the gene products of $597 \mathrm{bp}$ MIC10 were designed as forward: 5'-CCG GTC GAC ATG GCG CTT TCT TCT TTG AAC-3' and reverse 5'-CCG AAG CTT CTA CAT TGA TTT CCT GCG TC-3'. The underlined letters in the sequence of the primers represented the SalI and HindIII restriction enzymes linker sites in the 
expression vector pET32b (Novagen, Germany). The DNA was extracted from tachyzoites using DNA extraction kit (Qiagen) following the manufacturer's protocol and subjected to PCR which was performed as initial denaturation at $95^{\circ} \mathrm{C}$ for $5 \mathrm{~min}$, followed by 36 cycles of $95^{\circ} \mathrm{C}$ for $40 \mathrm{~s}, 59^{\circ} \mathrm{C}$ for $40 \mathrm{~s}, 72^{\circ} \mathrm{C}$ for $1 \mathrm{~min}$, with a final extension step at $72^{\circ} \mathrm{C}$ for $10 \mathrm{~min}$. The resulting gene product was ligated into the SalI and HindIII site of $\mathrm{pET} 32 \mathrm{~b}$ to generate a recombinant plasmid pET32b-MIC10 and processed for the expression of recombinant gene product in Escherichia coli BL21 codon plus competent cells according to the standard techniques of Sambrook and Russel (2001). The recombinant MIC10 was analyzed by SDS PAGE and the reactivity with Ni NTA anti histidine HRPase conjugate (Qiagen) in the western blot. The recombinant protein was purified using an Ni-NTA purification system (Qiagen, Germany) according to the manufacturer's protocol. The concentration of MIC10 protein was estimated as per the method described by Lowry et al., (1951) and using Nano Drop 1000 (Thermo Scientific Spectrophotometer, USA). The purified protein was kept at $-40^{\circ} \mathrm{C}$ till further use.

\section{Indirect enzyme linked immune sorbent assay}

The reactivity of expressed MIC10 protein was checked with $T$. gondii infected experimental sera from mice. ELISA was performed starting with coating of wells of microplates with the recombinant MIC10 antigen protein. A $100 \mu \mathrm{l}$ of the recombinant antigen (150 ng/well) suspended in carbonatebicarbonate buffer was coated onto the flat bottom 96 well ELISA plates (Nalgene Nunc Int., Germany) and incubated at $37^{\circ} \mathrm{C}$ for 2 hours. Then, the plates were washed thrice with PBS-T solution. Blocking was done with $1 \%$ Bovine serum albumin in PBS and incubated at $4{ }^{\circ} \mathrm{C}$ overnight. Subsequently, the plates were washed thrice with PBS-T and 100 $\mu \mathrm{l}$ of diluted experimental sera (1:20) samples were added in wells followed by incubation at $37^{\circ} \mathrm{C}$ for 1 hour. Then, the plates were again washed with PBS-T and $100 \mu \mathrm{l}$ of the diluted (1: 5000) rabbit anti-mouse IgG-HRPO conjugate (Sigma, Aldrich) was added to the wells and further incubated at $37{ }^{\circ} \mathrm{C}$ for 1 hour. Finally, the plates were washed thrice as earlier and each well was added with $100 \mu \mathrm{l}$ of the freshly prepared ELISA substrate solution (ortho phenylenediamine dihydrochloride), avoiding any direct exposure of the plate to light. The plates were kept in dark place at room temperature for 15 minutes and thereafter $50 \mu \mathrm{l}$ of stopping solution (2M $\mathrm{H}_{2} \mathrm{SO}_{4}$ ) was added to the wells and then optical density was measured at $492 \mathrm{~nm}$ using ELISA reader (Stat Fax-2100, Awareness Technology Inc.). The antigen, serum, conjugate and substrate controls were also included in the assays.

\section{Statistical analysis}

The data of ELISA were analyzed by Microsoft excel software and expressed in mean plus standard deviation (S.D). Sensitivity and specificity obtained from $\mathrm{TP} / \mathrm{TP}+\mathrm{FN} * 100$ and $\mathrm{TN} / \mathrm{TN}+\mathrm{FP} * 100$ formula respectively.

\section{Results and Discussion}

\section{Expression and purification of $T$. gondii MIC10}

The open reading frame of MIC10 encoding gene was amplified by PCR assay from the total genomic DNA obtained from tachyzoites of $T$. gondii $\mathrm{RH}$ strain and ligated into expression vector pET32b to construct recombinant plasmid pET32b-MIC10 and was transformed into E. coli BL21 codon plus cells with induction by IPTG for expression. The immune reactivity of the expressed protein 
was confirmed by western blot using the anti histidine HRPase conjugate. The purified rMIC10 expressed from its open reading frame (597 bp) have calculated molecular mass of $21.7 \mathrm{KDa}$. Since, the expression vector pET32b (Novagen, 2001) consists of thioredoxin tag (109 aa), his tag (6 aa) and $\mathrm{S}$ tag (15 aa) following the $\mathrm{T} 7$ promoter region with molecular weight of approximately 14.3 $\mathrm{KDa}$, the expressed protein obtained were sum of molecular mass from open reading frame and the tagged protein which was $36 \mathrm{KDa}$.

\section{Evaluation of diagnostic performance of MIC10}

The reactivity and potential of expressed recombinant MIC10 protein was checked with $T$. gondii infected and control group experimental sera from mice. Optimum working dilutions of the antigen, sera and the conjugate were determined by checker board titrations. From the stock concentration of recombinant protein of $150 \mu \mathrm{g} / \mathrm{ml}$, the optimum concentration of the MIC10 antigen for coating of plates for IgG ELISA was 150 ng per well. The optimum dilution of the antiIgG enzyme-labelled conjugate was 1 in 5000 and the optimum dilution of serum for $\mathrm{IgG}$ ELISA was found to be 1:20.

The 24 known positive and 39 known negative mice sera samples were tested for anti- $T$. gondii IgG antibodies by rec-MIC10-ELISA. The quantification was conducted through OD values for the 24 positive sera and 39 negative sera (Table 1). Out of 24 known positive sera, 19 showed positivity in rMIC-10 ELISA with five false negative. Out of 39 known negative sera samples, 34 showed negativity in rMIC10 ELISA with 5 false positive.

The cut off value in ELISA determined as average OD value of 39 negative controls plus standard deviation (S.D). The cut off value was calculated at 1.2539. After interpretation of data, $79.2 \%$ sensitivity and $87.2 \%$ specificity was shown by rMIC-10 ELISA. The indirect haemagglutination and latex agglutination in the present form have been considered as insensitive (Dubey et al., 1995).

The modified agglutination test (MAT), although specific, it is considered as time consuming and expensive, whereas ELISA, because of automation, has been largely accepted as the most practical test for diagnosis of toxoplasmosis (Dubey et al., 1995).

ELISA using native antigens of $T$. gondii tachyzoite is at present the most commonly used in serology for toxoplasmosis. However, it is well known that whole somatic antigenbased assay is difficult to standardize because such antigen preparation may contain extraparasitic components and hence may lead to interassay variability (Aubert et al., 2000).

Using a single or a combination of purified and defined proteins, including those derived from recombinant DNA technology, may be more reliable and specific (Aubert et al., 2000; Li et al., 2000; Pfrepper et al., 2005).

There are numerous ingenious test systems commercially available, which contain various tachyzoite antigen preparations that might be contaminated by extraneous parasitic material when passaged in mice or grown in vitro.

The serology results also may vary due to various antigen preparations used and interassay variability. Therefore, the need for purified standard reagents is greater today. Thus, recombinant $T$. gondii antigens can potentially replace crude tachyzoite material in toxoplasmosis serology. In the recent past, recombinant antigen based ELISA has emerged as the test of choice among existing serological techniques owing to its high sensitivity, specificity and potential for screening a large number of samples in a short period. 
Fig.1 Scatter diagram for OD values of known negative sera in mice (blue line depicting cut off value $=1.2539)$
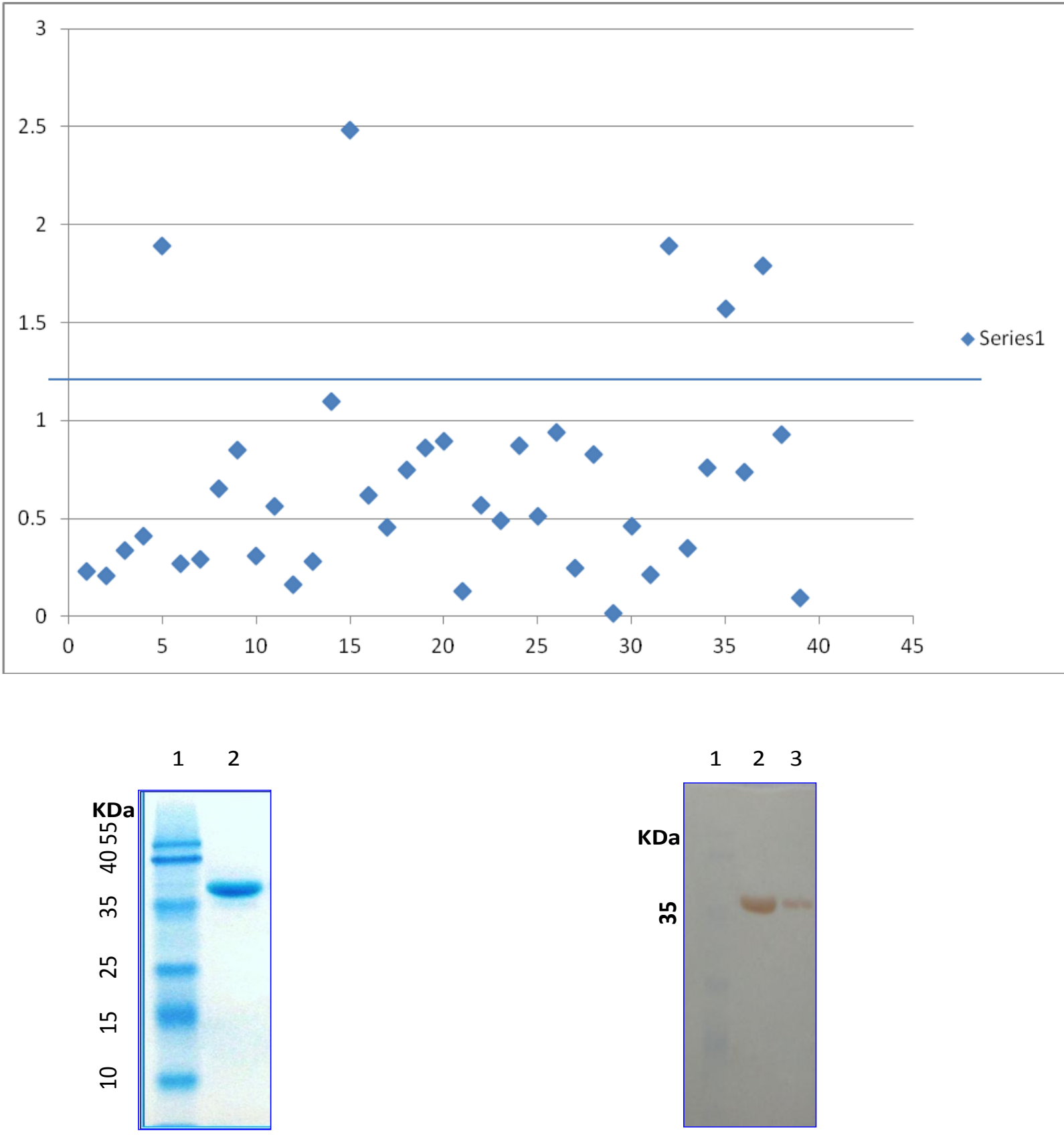

a) SDS-PAGE analysis of purified rMIC 10 protein of

T.gondii.

Lane 1: Prestained protein marker (Fermentas)

Lane 2: Purified recombinant MIC 10 protein
b)Western blot analysis of rMIC 10 protein of T.gondii

Lane 1: Prestained protein marker (Fermentas)

Lane 2\&3: Purified rMIC 10 protein of $T$. gondii 
Table.1 The OD values at $492 \mathrm{~nm}$ for known positive and negative sera of mice with evaluation of cut-off values for $T$. gondii

\begin{tabular}{|c|c|c|c|}
\hline S. NO & Known positive & & Known negative \\
\hline & OD value & & OD value \\
\hline 1 & 2.12 & & 0.23 \\
\hline 2 & 4.22 & & 0.21 \\
\hline 3 & 3.1 & & 0.34 \\
\hline 4 & 0.56 & & 0.412 \\
\hline 5 & 3.48 & & 1.89 \\
\hline 6 & 2.62 & & 0.27 \\
\hline 7 & 1.96 & & 0.29 \\
\hline 8 & 2.43 & & 0.653 \\
\hline 9 & 2.04 & & 0.851 \\
\hline 10 & 1.98 & & 0.312 \\
\hline 11 & 2.31 & & 0.56 \\
\hline 12 & 3.05 & & 0.164 \\
\hline 13 & 2.2 & & 0.281 \\
\hline 14 & 0.051 & & 1.1 \\
\hline 15 & 1.57 & & 2.48 \\
\hline 16 & 0.15 & & 0.619 \\
\hline 17 & 3.21 & & 0.458 \\
\hline 18 & 1.83 & & 0.751 \\
\hline 19 & 3.71 & & 0.862 \\
\hline 20 & 0.59 & & 0.893 \\
\hline 21 & 0.61 & & 0.127 \\
\hline 22 & 3.5 & & 0.57 \\
\hline 23 & 2.26 & & 0.492 \\
\hline 24 & 3.73 & & 0.87 \\
\hline 25 & & & 0.51 \\
\hline 26 & & & 0.94 \\
\hline 27 & & & 0.246 \\
\hline 28 & & & 0.825 \\
\hline 29 & & & 0.018 \\
\hline 30 & & & 0.46 \\
\hline 31 & & & 0.215 \\
\hline 32 & & & 1.89 \\
\hline 33 & & & 0.351 \\
\hline 34 & & & 0.762 \\
\hline 35 & & & 1.57 \\
\hline 36 & & & 0.74 \\
\hline 37 & & & 1.79 \\
\hline 38 & & & 0.93 \\
\hline \multirow[t]{4}{*}{39} & & & 0.096 \\
\hline & & Mean & 0.693 \\
\hline & & Std dev & 0.5609 \\
\hline & & Cut off & 1.2539 \\
\hline
\end{tabular}


A lot of recombinant antigens have been produced and used for development of ELISA for serodiagnosis of $T$. gondii infection in humans and animals including surface antigens (SAG1 and SAG2), rhoptry proteins (ROP2 and ROP4), microneme proteins (MIC1, MIC2, MIC3 and MIC4), and dense granule antigens (GRA1, GRA2, GRA5, GRA6, GRA7, and GRA8) (Pietkiewicz et al., 2004; Holec-Gasior 2013). We evaluated recombinant MIC10 for its diagnostic properties through known positive and known negative sera from murine model by indirect ELISA. The sensitivity and specificity data was generated suggesting that MIC10 is a good serological marker for detection of $T$. gondii infection and may be used for sero diagnosis in other species of animals and humans for identification of IgG antibodies. MIC10 is expressed by tachyzoites and is a circulating antigen (Hoff et al., 2001) could be available after invasion by tachyzoites for humoural response in the body of the host, making it a serological marker for the detection of anti $T$. gondii antibodies in acute infection. The present investigation is a step in the direction for exploring the use of microneme gene 10, molecular parasite target as a future tool for diagnosis. The cut-off was interpreted at 1.2539 with a moderate to good sensitivity and specificity $(79.2 \%$ and $87.2 \%$, respectively).

The diagnostic requirements and tools for toxoplasmosis vary greatly with the infected host species, state of infection and the physiological and immunological state of the human subjects like pregnancy, gestational age, immunocompetent versus immune compromised, etc. The source of the antigen usually for serology is the tachyzoite stage cultivated either in animal host or mammalian cell culture systems. The use of these undefined native antigens is often responsible for false-positve and false-negative reactions, because of the epitopes commonly shared between $T$. gondii and other closely related cyst-forming coccidia. The substitution of recombinant protein in place of tachyzoites has potential advantages in terms of cost, sensitivity, specificity, reproducibility, safety and commercial considerations. A number of parasite-specific proteins have been identified such as the membrane associated surface antigens (SAGs), dense granule antigens (GRAs), rhoptry proteins (ROPs), micronemal proteins (MICs), etc. and these candidate antigens have been shown to be useful for diagnosis as well as protection in experimental animals with attempts being made to achieve the best combination of immunoprophylactic / diagnostic antigens.

\section{Acknowledgement}

The authors acknowledge the department of Science and Technology, Govt. of India for funding this research and extend gratitude to PGIMER, Chandigarh for providing tachyzoites and TAH, IVRI, Mukteshwar for helping in conducting this study.

\section{References}

Aubert, D., Maine, G. T., Villena, I., Hunt, J. C., Howard, L., Sheu, M., Brojanac, S., Chovan, L. E., Nowlan, S. F. and Pinon, J. M. 2000. Recombinant antigens to detect Toxoplasma gondii-specific immunoglobulin $\mathrm{G}$ and immunoglobulin $\mathbf{M}$ in human sera by enzyme linked immunoassay. Journal of Clinical Microbiology, 38: 11441150.

Beghetto, E., Buffolano, W., Spadoni, A., Pezzo, M. D., Cristina, M. D., Minenkova, O., Petersen, E., Felici, F. and Gargano, N. 2003. Use of an immunoglobulin- $\mathrm{G}$ avidity assay based on recombinant antigens for diagnosis of primary Toxoplasma gondii Infection 
during pregnancy. Journal of Clinical Microbiology, 41: 5414-5418.

Dannemann, B. R., Vaughan, W. C., Thulliez, P. and Remington, J. S. 1990. Differential agglutination test for diagnosis of recently acquired infection with Toxoplasma gondii. Journal of Clinical Microbiology, 28: 1928-1933.

Dubey, J. P., Thulliez, P., Weigel, R. M., Andrews, C. D., Lind, P. and Powell, E. C. 1995. Sensitivity and specificity of various serological tests for detection of Toxoplasma gondii infection in naturally infected sows. American Journal of Veterinary Research, 56(8):1030-6.

Grigg, M. E. and Boothroyd, J. C. 2001. Rapid identification of virulent type I strains of the protozoan pathogen Toxoplasma gondii by PCR restriction fragment length polymorphism analysis at the B1 gene. Journal of Clinical Microbiology, 39: 398-400.

Haddadzadeh, H. R., Khazraiinia, P., Aslani, M., Rezaeian, M., Jamshidi. S., Taheri, M. and Bohonar, A. 2006. Seroprevalence of Toxoplasma gondii. Infection in stray and household cats in Tehran. Veterinary Parasitology, 138: 211-216.

Hoff, E. F., Cook, S. H., Sherman, G. D., Harper, J. M., Ferguson, D. J. P., Dubremetz, J, F. and Carruthers, V. B. 2001. Toxoplasma gondii: cloning and characterization of a novel $18-\mathrm{kDa}$ secretory antigen, TgMIC10. Experimental Parasitology, 97: 77-88.

Holec-Gasior, L. 2013. Toxoplasma gondii recombinant antigens as tools for serodiagnosis of human toxoplasmosis: current status of studies. Clinical and Vaccine Immunology 20: 1343-1351.

Kimbita, E. N., Xuenan, X., Xiaohong, H., Miyazawa, T., Fukumoto, S., Mishima, M., Suzuki, H., Sugimoto, C., Nagasawa, H., Fujisaki, K., Suzuki, N.,
Mikami, T. and Igarashi, I. 2001. Serodiagnosis of Toxoplasma gondii infection in cats by enzyme-linked immunosorbent assay using recombinant SAG1. Veterinary Parasitology, 102: 35-44.

Kotresha, D., and Noordin, R. 2010. Recombinant proteins in the diagnosis of toxoplasmosis. Acta Pathologica, Microbiologica, et Immunologica Scandinavica, 118: 529-542.

Li, S., Galvan, G., Araujo, F. G., Suzuki, Y., Remington, J. S. and Parmley, S. 2000. Serodiagnosis of recently acquired Toxoplasma gondii infection using an enzyme-linked immunosorbent assay with a combination of recombinant antigens. Clinical and Diagnostic Laboratory Immunology, 7: 781-787.

Lowry, O. H., Rosenbrough, W. J., Farr, A. L. and Randall, R. J. 1951. Protein measurement with the folin phenol reagent. The Journal of Biological Chemistry, 193: 265-275.

Marti, M., Li, Y., Köhler, P. and Hehl, A. B. 2001. Conformationally correct expression of membrane-anchored Toxoplasma gondii SAG1 in the primitive protozoan Giardia duodenali. Infectious Immunology, 70: 1014-1016.

Novagen. 2001. pET system manual. 11th ed. www.novagen.com.

Pfrepper, K. I., Enders, G., Gohl, M., Krczal, D., Hlobil, H., Wassenberg, D. and Soutschek, E. 2005. Seroreactivity to and avidity for recombinant antigens in toxoplasmosis. Clinical and Diagnostic Laboratory Immunology, 12: 977-982.

Pietkiewicz, H. E., Hiszczyn, S. E., Kur, J., Petersen, E., Nielsen, H. V., Stankiewicz, M., Andrzejewska, I. and Myjak, P. 2004. Usefulness of Toxoplasma gondii specific recombinant antigens in serodiagnosis of human toxoplasmosis. Journal of Clinical Microbiology, 42: 1779-1781. 
Sambrook, J. and Russel W. D. 2001. Molecular cloning: A laboratory manual. $3^{\text {rd }}$ edition. Cold spring harbor Laboratory press.

Velmurugan, G. V., Tewari, A. K., Rao, J. R., Baidya, S., Kumar, M. U. and Mishra, A. K. 2008. High-level expression of SAG1 and GRA7 gene of Toxoplasma gondii (Izatnagar isolate) and their application in serodiagnosis of goat toxoplasmosis. Veterinary Parasitology, 154: $185-192$.

Zhou, X. W., Kafsack, B. F. C., Cole, R. N. C., Beckett, P., Shen, R. F. and Carruthers, V. B. 2005. The opportunistic pathogen Toxoplasma gondii deploys a diverse legion of invasion and survival proteins. The Journal of Biological Chemistry, 280: 34233-34244.

\section{How to cite this article:}

Raghubir Singh, M.A. Malik, M. Sankar, Maninder Singh, H.K. Sharma and Sahil, K. 2018. Development of an Indirect Enzyme-Linked Immunosorbent Assay Using Recombinant Microneme 10 (MIC10) Protein of Toxoplasma gondii for Diagnostic Evaluation in Murine Model Infected with Tachyzoites. Int.J.Curr.Microbiol.App.Sci. 7(03): 416-425. doi: https://doi.org/10.20546/ijcmas.2018.703.049 